\title{
Mixed copolymer adlayers allowing reversible thermal control of single cell aspect ratio
}

F. Dalier, ${ }^{a}$ G. V. Dubacheva, ${ }^{a \dagger}$ M. Coniel, ${ }^{a}$ D. Zanchi, ${ }^{a, d}$ A. Galtayries, ${ }^{b}$ M. Piel, ${ }^{c}$ E. Marie, ${ }^{*}{ }^{, a}$ C. Tribet ${ }^{*}, a$.

${ }^{a}$ PASTEUR, Dept. Chimie, Ecole Normale Supérieure, PSL Research University, Sorbonne Universités, UPMC Univ. Paris 06, CNRS, 75005 Paris, France

${ }^{\mathrm{b}}$ PSL Research Univ., Chimie ParisTech, CNRS, Inst Rech Chim Paris, F-75005 Paris, France.

${ }^{\mathrm{c}}$ PSL Research Univ., CNRS UMR 144, Inst Curie, F-75005 Paris, France

${ }^{\mathrm{d}}$ Université de Paris 7 Denis Diderot, 5 rue Thomas Mann, 75013 Paris, France

Keywords: cell polarization $\bullet$ cell adhesion $\bullet$ poly(lysine $) \cdot \operatorname{poly}(\mathrm{N}$-isopropylacrylamide $) \cdot$ peptide display. 
ABSTRACT. Dynamic guidance of living cells is achieved by fine tuning and spatiotemporal modulation on artificial polymer layers enabling reversible peptide display. Adjustment of surface composition and interactions is obtained by coadsorption of mixed poly(lysine) derivatives, grafted with either repellent PEG, RGD adhesion peptides, or $T$-responsive poly(Nisopropylacrylamide) strands. Deposition of mixed adlayers provides a straightforward mean to optimize complex substrates, which is here implemented to achieve 1) thermal control of ligand accessibility and 2) adjustment of relative adhesiveness between adjacent micropatterns, while preserving cell attachment during thermal cycles. The reversible polarization of HeLa cells along orthogonal stripes mimics guidance along natural matrices. 
Cells do not live in a static environment. They continually sample the changes in their vicinity and adapt accordingly their behavior. For in vitro cell cultures, there is thus a growing demand to mimic the natural time-modulated cell:substrate interactions. Introduction of a time-controlled switch has for instance opened key perspectives in tissue engineering ${ }^{1}$ as it enables sequential deposition of cells, triggered detachment for cell "harvesting", and guidance by chemotaxies or mechanotaxies. ${ }^{2},{ }^{3}$ Dynamic control on artificial substrates would also enable studies of key determinants of stem cell fate.

A general approach relies on spatiotemporal control of the accessibility of specific molecular cues on culture substrates. The design of "molecular tools", typically macromolecular layers, enabling in situ controls on the presentation and/or binding of small molecules such as peptides, is thus one of the key issue in the field of artificial bio-interfaces. ${ }^{4}$ On one hand, "one-shot" irreversible switch can be obtained either by covalent attachment of adhesion molecules, or by specific cleavage. Typically, on demand cell attachment (resp. full detachment and harvesting) is achieved by coupling adhesion peptides onto cell-repellant patterns, e.g. via click chemistries ${ }^{56}$, (resp. via cleavage of peptides, e.g. photo-cleavage ${ }^{7,8,9}$ ). Compared to cultures on preconditioned static substrates, even this single in situ on/off switch has important consequences on cell fate (e.g. different gene expression pattern depending on the presentation time of peptides ${ }^{10}$ ). On the other hand, reversible modulation can be obtained by application of stimuli, including exposition to light, ${ }^{11,12}$ redox potential, ${ }^{13,14}$ or variation of temperature $(T) .{ }^{15}$ A predominant and robust approach is based on $T$-responsive layers, ${ }^{16},{ }^{17},{ }^{18}$ among which poly(N-isopropylacrylamide) (PNIPAM)-based coatings have reached a remarkable level of technological developments as it allows mild cell harvesting in a broad diversity of cell lines ${ }^{19},{ }^{18},{ }^{20}$ up to in vivo control on wound healing. ${ }^{21}$ Moreover, peptide-modified PNIPAM provide targeting of specific cell receptors. ${ }^{15}$ 
Reported implementations of thermal switch are however far to mimic cell guidance on natural substrates as they cannot keep cells adherent at all time. ${ }_{,, 2}^{1,22}$ Due to the broad variety of substrates, diversity of culture conditions and cell line, the major challenge in this field is to provide an easy-to-implement, non-toxic, versatile technology enabling subtle tuning by nonchemists of cell:substrate interaction.

To achieve this goal, which can be illustrated by on demand orientations of cell polarization or migration patterns, we propose here a straightforward surface preparation approach, based on adsorption of comb-like copolymers as mixed layers. Mixed layers enable to balance repellency, thermal modulation, and specific peptide binding (Scheme 1). We relied on polycationic derivatives of poly(lysine) (PLL) that spontaneously adsorb onto most substrates used in biology. PLL-containing layers have proved to be efficient biocompatible "glues"23 and their PEGylated derivatives (PLL-g-PEG) are known to form biorepellent adlayers. ${ }^{24}$ Here, PNIPAM and RGDmodified PNIPAM chains were introduced in the PLL backbone to obtain $T$-responsive and peptide-presenting chains. The deposition, as mixed adlayers, of PLL copolymers and $T$ triggered collapse of PNIPAM strands were assessed by quartz crystal balance (QCM-D). Thermal control of the adhesion and deadhesion of HeLa cells was adjusted by varying surface density of RGD-containing PNIPAM so that we could reach optimized layer compositions providing reversible control on competitive cell spreading onto different micropatterned areas (prepared by UV etching), while keeping the cells adherent at all time. This control is reminiscent of cell guidance in extracellular matrix, as driven by variation of the presentation of natural molecular cues. 

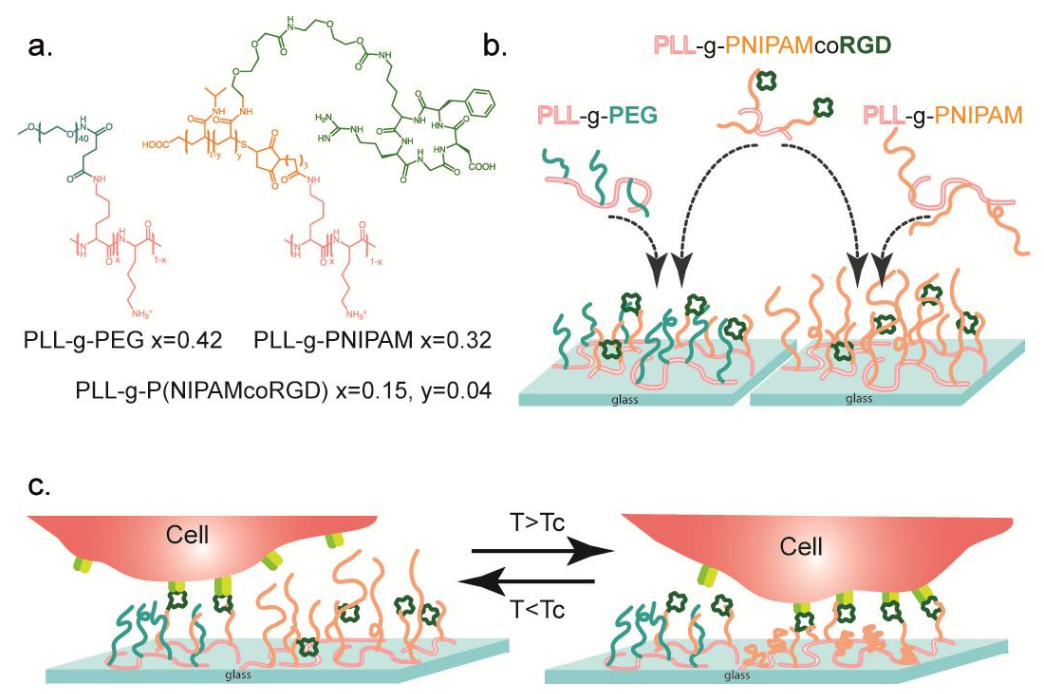

Scheme 1. Structures of comb-like PLL copolymers and their mixed T-responsive adlayers used to reversibly modulate the specific and preferential cell adhesion onto adjacent micropatterns. a) copolymers structures with $\mathrm{x}$ the mol\% grafting of PLL backbone; not shown the PLL-gPNIPAM is the same as PLL-g-PNIPAMcoRGD, but with isopropylamine side groups only and no RGD. b) representation of mixed adlayers formed by spontaneous co-adsorptions. c) scheme of temperature-modulated peptide dispaly and control of binding of cell's receptors.

Scheme 1 shows PNIPAM-presenting copolymers, PLL-g-P(NIPAMcoRGD) and PLL-gPNIPAM, resp. with and without 4 mol\% cyclic RGD attached on the PNIPAM strands. They were synthesized by slight modifications of a reported procedure, by grafting NHS-terminated PNIPAM chains to a PLL precursor chain ${ }^{25}$ (see synthesis detailed as Supporting Information). The adsorption of polycationic comb-like PLL-g-PNIPAM was performed on a plasma-cleaned, or $\mathrm{NaOH}$ pre-conditioned, anionic silica surfaces. The decrease in resonance frequency, $\Delta f$, observed by QCM-D measurements on bare silica-coated quartz sensors flushed with a $1 \mathrm{~g} / \mathrm{L}$ solution of PLL-g-PNIPAM was indicative of fast deposition of polymers, a phenomenon that 
essentially took place within $30 \mathrm{~s}$ (Fig. S1). Flushing out the polymer excess and rinsing with water did not significantly affect the adsorbed mass as indicated by variations of $\Delta f$ in Figure 1 .

To assess the $T$-triggered transition in adlayers, the polymer-coated quartz sensors were subjected to stepwise $T$ sweeps, while being in contact with polymer-free PBS buffer. Representative variations of frequency and dissipation for the sensor functionalized with PLLPNIPAM are shown in Figure 1. The maximum rate of variation of $\Delta f$ and $\Delta D$ were detected at $T$ $=32^{\circ} \mathrm{C}$ and $30^{\circ} \mathrm{C}$ respectively (Fig. S2), suggesting that the surface layer undergoes a transition at about the LCST of bulk PNIPAM solutions. Reversibility of the transition was indicated by the superimposition of signal traces recorded during upward and backward variations of $T$ and by repeatable plateaus reached upon cycling between $25^{\circ} \mathrm{C}$ and $32^{\circ} \mathrm{C}$ (Fig. S3). Remarkably, the increase in $\Delta f$ (resp. decrease in $\Delta D$ ) above $T=32^{\circ} \mathrm{C}$ is indicative of a mass loss (resp. a rigidification) that is consistent with collapse and dehydration of PNIPAM strands, and is consistent with increased hydrophobicity at high temperature as shown by previous AFM forcedistance curve estimates on similar PLL-g-PNIPAM layers. ${ }^{25}$ In contrast, measurements performed on silica coated with PLL-g-PEG or PLL-g-P(NIPAMcoRGD) (Fig. S2 \& S4) indicate the absence of collapse in both PEG and RGD-modified PNIPAM layers in the same experimental window. Lack of transition of RGD-modified PNIPAM suggests that RGD increases markedly the hydrophilicity of PNIPAM chains. 

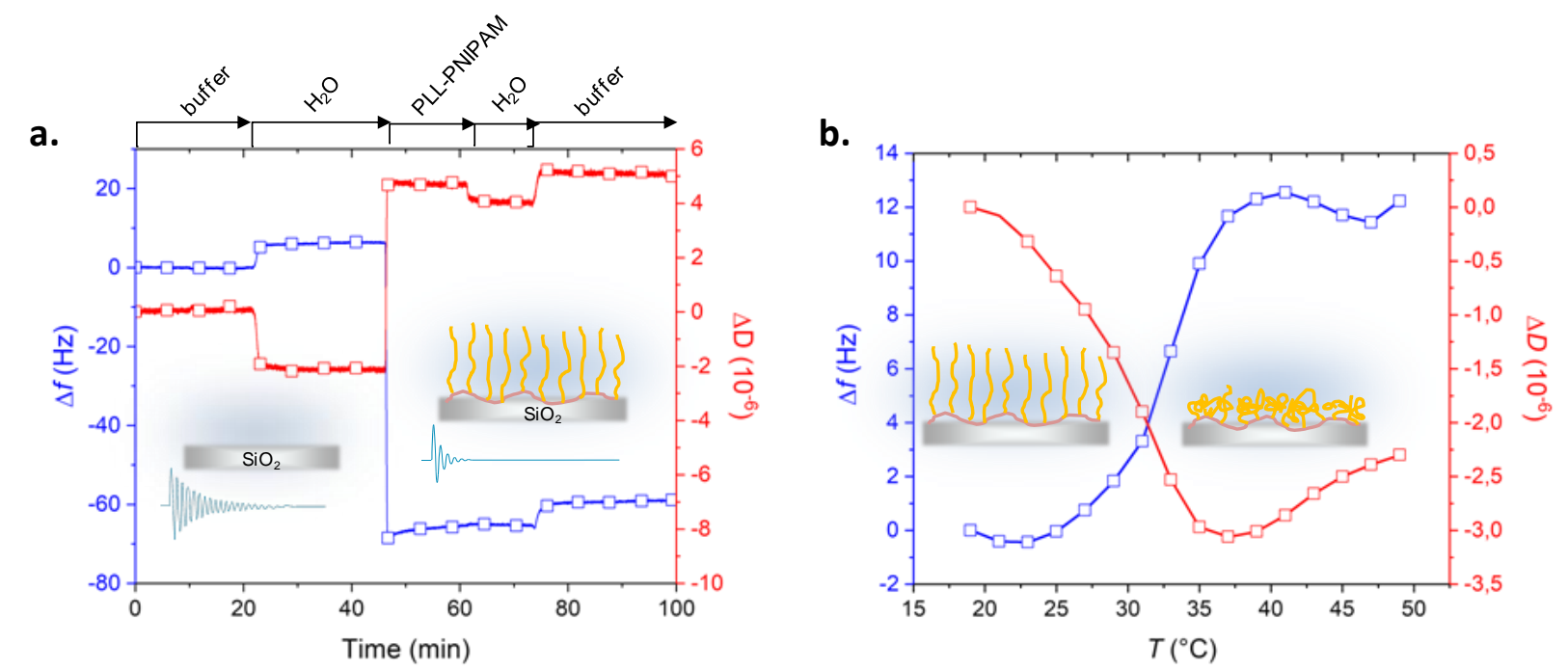

Figure 1. QCM-D monitoring of PLL-PNIPAM binding. Variations of $\Delta f$ and $\Delta D$ observed upon a) flushing $(150 \mu \mathrm{L} / \mathrm{min})$ aqueous solutions at $25^{\circ} \mathrm{C}(1 \mathrm{~g} / \mathrm{L}$ PLL-g-PNIPAM in water); b) increasing $T$ in PBS, in the absence of polymer in buffer (N.B. an offset and the thermal drift measured on uncoated bare silica were substracted to raw data; PBS buffer is $10 \mathrm{mM}$ sodium phosphate $\mathrm{pH} 7.4,150 \mathrm{mM} \mathrm{NaCl}$ ). Insets show silica surface with swollen or collapsed chains, and piezoelectric response before and after polymer adsorption.

The binding of mammalian cells onto PLL-g-P(NIPAMcoRGD) was assessed on layers formed by a bath application of mixed solutions of PLL derivatives. As shown in previous studies, ${ }^{25,6,26}$ adsorption from a mixed solution of two comb-like PLL derivatives yields a mixed adlayer whose composition reflects the solution one. Adhesion of cells on layers of decreasing densities of RGD was accordingly explored on glass coverslips coated by mixed solutions of PLL-g-P(NIPAM-co-RGD):PLL-g-PNIPAM at varying molar $\%, \quad \tau$, of RGD-containing copolymer (see Supportings for details). HeLa cells were seeded at a density of $5.10^{3} \mathrm{cells} / \mathrm{cm}^{2}$ on coverslips preequilibrated in serum-free DMEM, at either 27 or $37^{\circ} \mathrm{C}$. Cell areas and high/low birefringence were criteria used to distinguish "round" highly birefringent, unbound, cells from spread, weakly birefringent, adherent ones (Fig.2a). The cells typically needed 1.5 - 2 
$\mathrm{h}$ to reach equilibrium spreading (Fig. S5 in Supportings). On the layers of low RGD densities (i.e. $\tau<7 \mathrm{~mol} \%$ PLL-g-P(NIPAMcoRGD)), the plateau fraction of spread cells decreased markedly (Fig. 2). Finally, a negligible number of cells was found to spread on 100\% PLL-gPNIPAM, indicating the high repellency achieved at time $<3 \mathrm{~h}$ in the absence of RGD, both at $27^{\circ} \mathrm{C}$ and $37^{\circ} \mathrm{C}$ (Fig 2 and S5a, data at $0 \%$ RGD). This suggests that spreading essentially occurs due to specific binding onto RGD-displaying copolymers. The non specific binding that is known to enable cell culture on substrates covered at high T by PNIPAM-adsorbed proteins, ${ }^{15,19}$ is not an issue in the present conditions. Based on the ratio of spread/round cells, $\rho$, reached at time 180 min. after cell seeding, we estimated that the range of $5-20 \%$ provided the optimal effective RGD densities needed to achieve thermal responses.

a.
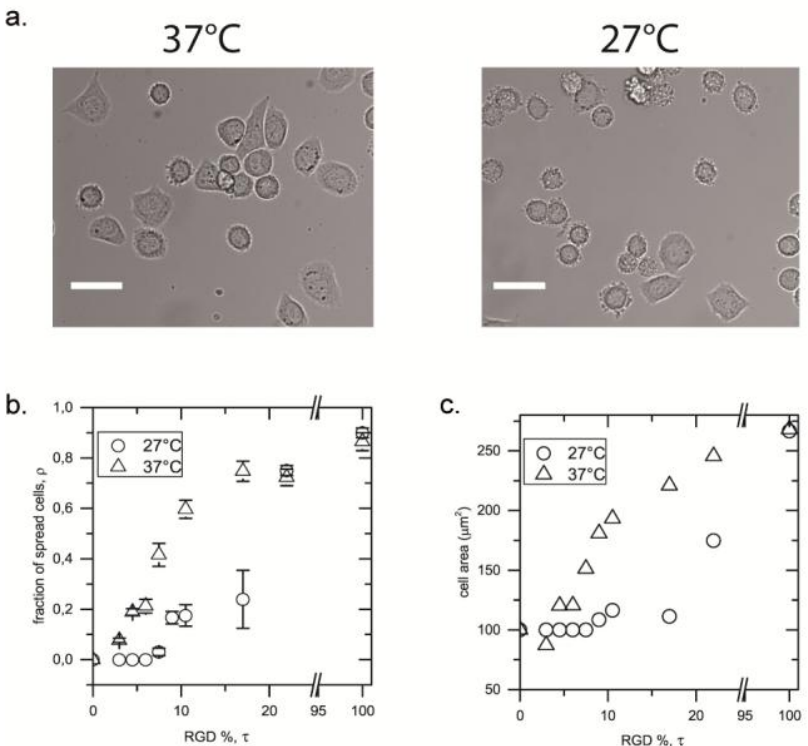

Figure 2. Effect of $T$ on adhesion of HeLa cells onto PLL-g-PNIPAM:PLLg-P(NIPAMcoRGD) mixed layers. a) Representative microscopy phase contrast images on coverslip precoated with a mixture at $10 \mathrm{~mol} \%$ PNIPAMcoRGD; cells were deposited and incubated either at $37^{\circ} \mathrm{C}$ or $27^{\circ} \mathrm{C}$; scale bar $50 \mu \mathrm{m} . \mathrm{b}$ ) Variation of the degree of spreading and c) average area per cell seeded on coverslips precoated with PLL-g-PNIPAM:PLL-g-P(NIPAMcoRGD) solutions of 
increasing molar fraction, $\tau$, of the RGD-containing copolymer. Measurements were carried out at time $3 \mathrm{~h}$ after cell deposition, in serum-free DMEM.

On coverslips coated with PLL-g-P(NIPAMcoRGD):PLL-g-PNIPAM, a sharp increase of the spreading index $\rho$ was detected above a threshold $\tau$ of a few $\%$, which is indicative of a minimum density of RGD needed for adhesiveness. ${ }^{27}$ This threshold was upshifted at $T=27^{\circ} \mathrm{C}$ as compared to $37^{\circ} \mathrm{C}$ (Fig. 2b). The average cell areas essentially reflected similar trends as the spread/round ratios (Fig. 2c). These results suggest that the collapse/swelling transition of PLLg-PNIPAM in the adlayer affects accessibility of RGD. As a control of the absence of cell sensitivity to $T$, we checked the absence of thermal response on pure PLL-g-P(NIPAMcoRGD) $(\tau=100 \%)$ lacking the collapse transition.

Next, we assessed the effect of layer composition on cell detachment upon cooling from $37^{\circ} \mathrm{C}$ down to $27^{\circ} \mathrm{C}$. Isolated cells were deposited on adhesive mixed layers, at $T=37^{\circ} \mathrm{C}$, and incubated for $3 \mathrm{~h}$ to provide enough time for the formation of a population of predominantly spread cells. The culture wells were then cooled down to $27^{\circ} \mathrm{C}$, and the fraction of cells that detached, i.e. turned from spread into round shapes was measured (Fig. 3, supporting video 1). As expected, no detachment occurred on $100 \%$ PLL-g-P(NIPAM-co-RGD) that do not undergo thermal transition. At lower $\tau(<10 \%)$ in contrast, round shape cells gradually formed at the expense of the spread cell population. Reversion of adhesiveness at low $T$ is thus achieved in a limited window of mixed layer composition that fulfill the requirements of 1) presenting a density of accessible RGD above adhesion threshold, 2) containing a predominant fraction of RGD-free, $T$ responsive PLL-g-PNIPAM. Deadhesion at low $T$ was rapid at the level of a single cell (ca. 10 
min., see video) which is presumably a signature of using thin PNIPAM layers. ${ }^{22}$ The average incubation time required for full detachment, as reflected by variations of the mean $\%$ of spread cells (about 100 min. in Fig. 3a), reflects accordingly more a cell-to-cell variability of the onset of the response to $\mathrm{T}$, which is not surprising in a population containing cells at different stage of their natural cycle. An important result here is the identification of optimal layer compositions that allow controlled attachment/detachment. Of note, cell detachment was efficient on $9 \%$ RGD coatings ( $>90 \%$ round cells at $\mathrm{t}=200 \mathrm{~min}$.) whereas no detachment was observed on $16 \%$ RGD coatings. The spontaneous PLL adsorption enables efficient adjustment of the optimal ratio between cell adhesive and $T$-switchable copolymers. Furthermore, using PLL-g-PNIPAM devoid of RGD as a trigger keep the sharp transition and threshold $T$ unmodified irrespective of RGD densities.

a.

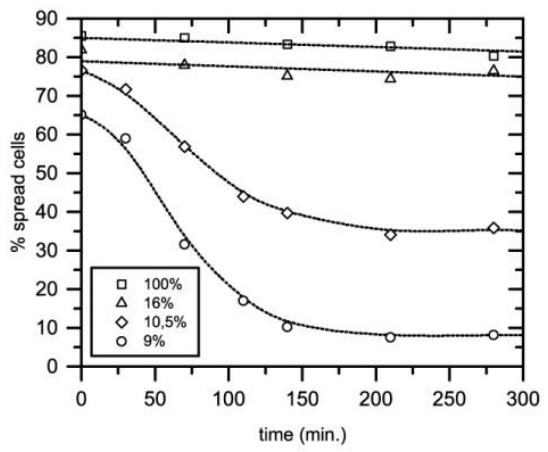

b.
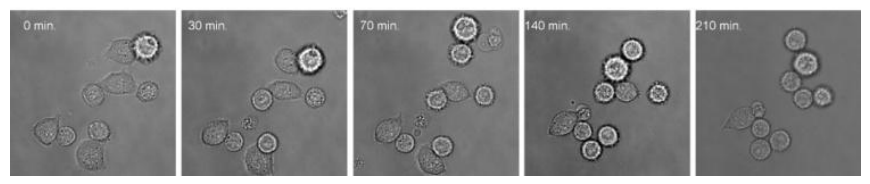

Figure 3. Cell detachment triggered upon cooling adherent isolated HeLa cells from $37^{\circ} \mathrm{C}$ down to $27^{\circ} \mathrm{C}$. a) Time variation of the ratio between spread and round cells on layers of varying composition (\% PNIPAMcoRGD in the preconditioning solution is quoted in figure), cells were 
preincubated for $3 \mathrm{~h}$ at $37^{\circ} \mathrm{C}$ in DMEM prior to decreasing $T$ down to $27^{\circ} \mathrm{C}$ at time zero. b) Representative phase contrast images of cells on a coverslip coated at 9 mol\% PLL-gP(NIPAMcoRGD).

Finally, to mimic the dynamic guidance of cells, we tailored the layer compositions of adjacent areas. The goal was to specifically and reversibly orient cell spreading and polarization along orthogonal axes. We observed thermal response of HeLa cells deposited at cross points of two microstripes, one being covered with both adhesive and $T$-responsive layer, the other one being coated with an adhesive, non-responsive layer (Fig. 4a). The pattern was prepared by coating glass coverslips with PLL-g-PEG as a repellent continuum, followed by UV-etching micrometerlarge parallel stripes onto which a mixed PLL-g-PNIPAM:PLL-g-P(NIPAM-co-RGD) was adsorbed ( $\tau$ between $7.7 \%$ and $10.5 \%$ ). Orthogonal non-responsive microstripes were then UVetched and covered with PLL-g-PEG:PLL-g-P(NIPAMcoRGD) mixed layer ( $\tau$ between $4 \%$ and $16 \%$ ). Adjustment of the \%RGD in both stripes was key to obtain a significant fraction of cells adopting cross-like or lozenge shapes and to balance the adhesion of cells onto each stripe. For instance, when the PEGylated stripes contained less than 6\%PNIPAMcoRGD a majority of cells were migrating along PNIPAM-based stripes (at 9\% PNIPAMcoRGD). Conversely, PEGylated stripes prepared with 16\% PNIPAMcoRGD became the predominant sites for cell binding and elongation, at the expense of PNIPAM-based stripes prepared below $7 \mathrm{~mol} \%$ PLL-gP(NIPAMcoRGD). Well balanced compositions was thus required to obtain that a significant fraction of cells bind at the cross-points between orthogonal stripes. To this end, we fixed to $16 \%$ the fraction of PNIPAMcoRGD mixed with PLL-g-PEG. In these conditions, cells elongated toward both orthogonal directions and adopted a lozenge shape (Fig.4b). 


$$
\text { a. }
$$
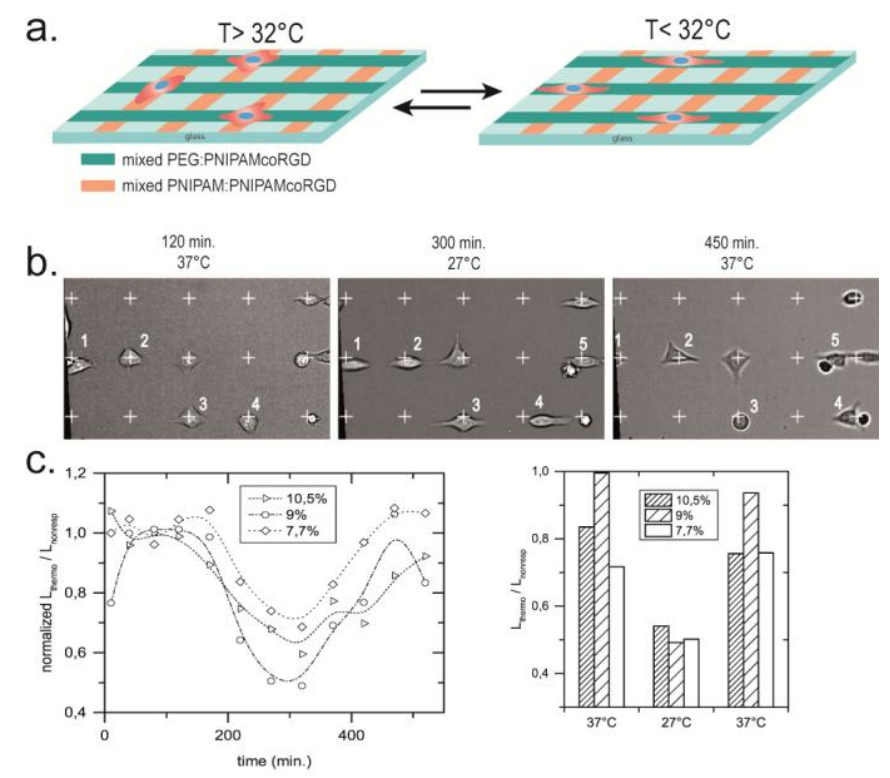

Figure 4. Thermal control of competitive cell elongation along orthogonal microstripes of wellbalanced RGD densities. a) Scheme of the micropatterns with cells sitting at cross point of $T$ responsive stripes (PNIPAM:PNIPAMcoRGD) and non-responsive ones (PEG:PNIPAMcoRGD). b) Phase contrast images of cells; white crosses indicate the cross points between horizontal (non-responsive) and vertical ( $T$-responsive) stripes; numbers ( 1 to 5 ) are quoted to point on a few representative cells, that during the thermal cycle either reversibly change from lozenge shape to elongated one (cells 1,2, 4 \& 5) and/or migrate in (cell \#5) or out (cell\#1) the filed of view; cell \# 3 became round as it underwent a division at time $>450 \mathrm{~min}$. c) Ratio of cell lengths along each directions $L_{\text {thermo }} / L_{\text {nonresp }}$ averaged over a population of about 30 cells all sitting at cross points between stripes during the whole cycle. Thermal cycle was $120 \mathrm{~min}$. at $37^{\circ} \mathrm{C}, 200 \mathrm{~min}$. at $27^{\circ} \mathrm{C}$, and finally back to $37^{\circ} \mathrm{C}$. \% PNIPAM-coRGD is quoted in inset.

Temperature was cycled to assess cell's propensity for spreading and extending along the $T$ switchable stripe vs the non-responsive ones. After seeding $\left(3 \mathrm{~h}\right.$ in serum-free DMEM at $\left.37^{\circ} \mathrm{C}\right)$, cells sitting at the cross-points between vertical and horizontal stripes were selectively observed. Cells were then subjected to two successive $T$ jumps, first from 37 down to $27^{\circ} \mathrm{C}$ at time 120 
minutes, and second from 27 up to $37^{\circ} \mathrm{C}$ at time 320 minutes. Upon cooling down to $27^{\circ} \mathrm{C}$, retraction from the thermo-responsive areas (orange or vertical axes in Fig. 4a,b) occurred in parallel to cell extension along the orthogonal PEGylated stripes. Upon increasing $T$ back to 37 ${ }^{\circ} \mathrm{C}$, cells elongated along the $T$-responsive stripe, while retracting from the PEGylated one (see videos $2 \& 3$ in Supporting). In general, no cell detachment occurred during these transitions. To evaluate the cell polarity we measured cell lengths along the PLL-g-PNIPAM-containing stripes, $L_{\text {thermo }}$, and the PEG-containing ones, $L_{\text {nonresp }}$. Time and/or $T$ variation of $L_{\text {thermo }} / L_{\text {nonresp }}$ (averaged over a population of $20-40$ cells that were all sitting at cross points and not migrating neiher dividing during the whole cycle, i.e. excluding cells such as the one quoted $3 \& 4$ in Fig. 4 b) are shown in Fig. 4c. A few minutes after cooling, the cells preferred to spread along PEGylated stripes. Similar to the fast detachment in response to $T$ observed on homogeneous substrates (Fig. $3 b)$, fast $(<10 \mathrm{~min}$.) individual reorganisation but high cell to cell variability of the onset time of the response was observed for cell spread along stripes (video $2 \& 3$ ). The population-averaged polarity change as shown in Fig. $4 \mathrm{c}$ and the time of plateauing of the elongation ratio (about $1 \mathrm{~h}$ incubation irrespective of $\tau$ ) is thus reflecting the natural population variability and a broad range of the delay of response to stimulation. Of note, polarity switch was slightly more important at 9\% RGD (Fig.4c right, compared to $10.5 \%$ and $7.7 \%$ ) suggesting that competition with the nonresponsive stripe is optimized by adjustment of the thermo-responsive one.

\section{Conclusion.}


The above observations suggest that PLL-g-PNIPAM, and its peptide-modified derivative are useful tools to tune peptide densities and achieve control display of peptides on cell culture substrates. Spontaneous adlayer formation by mixtures of PLL derivatives enables fine tailoring of compositions thus allowing to guide cell polarity and to mimic ECM dynamic remodeling. The straightforward, benchtop procedure for surface coating is amenable to micropatterning, and can be implemented on most common substrates (glass, PDMS, polystyrene ${ }^{26}$ ) including nonplanar ones (e.g. colloid particles ${ }^{28}$ ), which offers to biologists a broad space of parameters to explore, including the possibility to codeposit polymer strands of different chemical natures in the same layer (e.g. PEG and PNIPAM ${ }^{28}$ ). Possible applications include on demand guidance of cell growth and migration, stimulation of a subpopulation of cells in the culture (e.g. controlled co-culture), and studies of the rate of cell attachment or migration in response to local activation (e.g. to trigger and study stem cell differentiation, cancer cell migration, etc). Dynamic interfaces allow to go one step further in the development of artificial scaffolds. As opposed to reported layers aiming at full cell detachment and cell sheet harvesting, mixed layers of PLL-gP(NIPAM-co-peptides) makes possible more flexible adjustment of the balance between nonspecific repellency and specific adhesion, with temporal variations which is key to explore behavior of permanently adherent cells. These conditions partially recapitulate the attributes observed in a natural environment.

\section{ASSOCIATED CONTENT}

Supporting Information. Procedures for synthesis and characterization of the polymers, QCM-D data on adsorption and thermal response of polymer adlayers, materials and methods used for Hela cells spreading on polymer adlayers (coatings, cell seeding, micropatterning by UV-etching) are supplied as Supporting Information. 
The following movies are available free of charge.

Two representative examples of Hela cells deposited at cross points between orthogonal $T$ responsive and non-responsive stripes and exposed to $T$ variation as quoted in the videos: video 1 \& video 2 (file type, AVI),

\section{AUTHOR INFORMATION}

\section{Corresponding Author}

*emmanuelle.marie@ens.fr.* christophe.tribet@ens.fr.

\section{Present Addresses}

† PPSM CNRS UMR 8531, ENS Cachan, Université Paris-Saclay, 61 Avenue du Président Wilson, 94235 Cachan, France.

\section{Author Contributions}

The manuscript was written through contributions of all authors. All authors have given approval to the final version of the manuscript.

\section{ACKNOWLEDGMENT}

Work was supported by ANR DAPPlePur 13-BS08-0001, and Labex “Dynamo” ANR-11LABX-0011-01 to C.T. and E.M.

\section{ABBREVIATIONS}

PLL, poly(lysine) ; PNIPAM, poly(N-isopropylacrylamide); PEG, poly(ethyleneoxyde); RGD, Arg-Gly-Asp-Phe-Lys cyclic polypeptide side group. 
SYNOPSIS

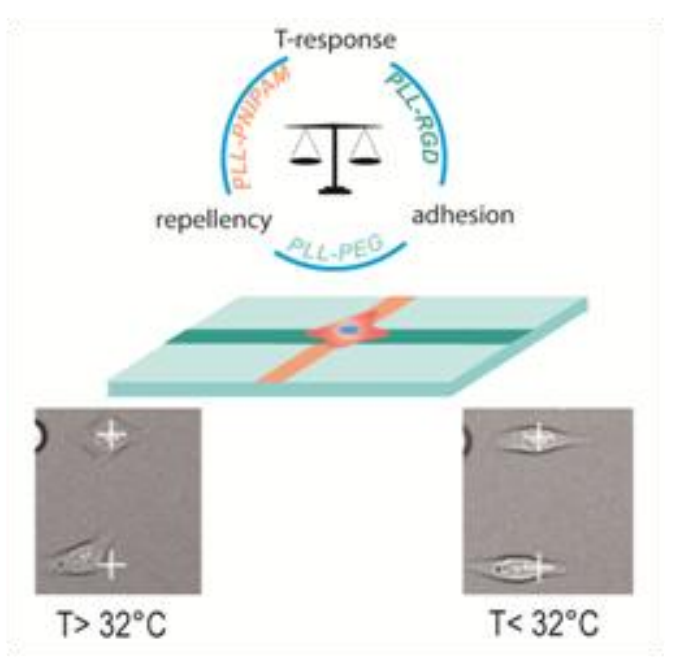

Finding the right balance. control of specific cell adhesion is achieved via ubiquitous adsorption of comb-like poly(lysine) derivatives from mixtures of biorepellent, bioadhesive, thermally switchable chains. At balanced repellency vs adhesiveness, the thermal stimulation reversibly guides cell's polarization along micropatterns without detachment, which mimics cell guidance on natural matrices. This method accessible to non-chemists enables straightforward, benchtop optimization of functional mixed layers.

\section{REFERENCES}

1. Kim, J.; Hayward, R. C., Mimicking dynamic in vivo environments with stimuliresponsive materials for cell culture. Trends Biotechnol. 2012, 30 (8), 426-439.

2. Skorb, E. V.; Andreeva, D. V., Surface Nanoarchitecture for Bio-Applications: SelfRegulating Intelligent Interfaces. Adv. Funct. Mater. 2013, 23 (36), 4483-4506.

3. Hribar, K. C.; Choi, Y. S.; Ondeck, M.; Engler, A. J.; Chen, S. C., Digital Plasmonic Patterning for Localized Tuning of Hydrogel Stiffness. Adv. Funct. Mater. 2014, 24 (31), 49224926. 
4. $\quad$ Higuchi, A.; Ling, Q. D.; Kumar, S. S.; Chang, Y.; Kao, T. C.; Munusamy, M. A.; Alarfaj, A. A.; Hsu, S. T.; Umezawa, A., External stimulus-responsive biomaterials designed for the culture and differentiation of ES, iPS, and adult stem cells. Prog. Polym. Sci. 2014, 39 (9), 1585-1613.

5. $\quad$ Schmitt, S.; Huemmer, J.; Kraus, S.; Welle, A.; Grosjean, S.; Hanke-Roos, M.; Rosenhahn, A.; Braese, S.; Woell, C.; Lee-Thedieck, C.; Tsotsalas, M., Tuning the Cell Adhesion on Biofunctionalized Nanoporous Organic Frameworks. Adv. Funct. Mater. 2016, 26 (46), 8455-8462.

6. $\quad$ van Dongen, S. F. M.; Maiuri, P.; Marie, E.; Tribet, C.; Piel, M., Triggering Cell Adhesion, Migration or Shape Change with a Dynamic Surface Coating. Adv. Mater. 2013, 25 (12), 1687-1691.

7. Nakanishi, J., Switchable Substrates for Analyzing and Engineering Cellular Functions. Chem.- Asian J. 2014, 9 (2), 406-417.

8. Rolli, C. G.; Nakayama, H.; Yamaguchi, K.; Spatz, J. P.; Kemkemer, R.; Nakanishi, J., Switchable adhesive substrates: Revealing geometry dependence in collective cell behavior. Biomaterials 2012, 33 (8), 2409-2418.

9. Wang, X. Z.; Yao, C.; Weng, W. J.; Cheng, K.; Wang, Q., Visible-Light-Responsive Surfaces for Efficient, Noninvasive Cell Sheet Harvesting. Acs Appl. Mater. Inter. 2017, 9 (34), $28250-28259$.

10. Lee, E. J.; Luo, W.; Chan, E. W. L.; Yousaf, M. N., A Molecular Smart Surface for Spatio-Temporal Studies of Cell Mobility. Plos One 2015, 10 (6): e0118126.

11. Bian, Q.; Wang, W. S.; Wang, S. T.; Wang, G. J., Light-Triggered Specific Cancer Cell Release from Cyclodextrin/Azobenzene and Aptamer-Modified Substrate. Acs Appl. Mater. Inter. 2016, 8 (40), 27360-27367.

12. Gandavarapu, N. R.; Azagarsamy, M. A.; Anseth, K. S., Photo-Click Living Strategy for Controlled, Reversible Exchange of Biochemical Ligands. Adv. Mater. 2014, 26 (16), 25212526.

13. Ng, C. C. A.; Magenau, A.; Ngalim, S. H.; Ciampi, S.; Chockalingham, M.; Harper, J. B.; Gaus, K.; Gooding, J. J., Using an Electrical Potential to Reversibly Switch Surfaces between Two States for Dynamically Controlling Cell Adhesion. Angew. Chem.-Int. Ed. 2012, 51 (31), 7706-7710.

14 Yeung, C. L.; Wang, X. Y.; Lashkor, M.; Cantini, E.; Rawson, F. J.; Iqbal, P.; Preece, J. A.; Ma, J.; Mendes, P. M., Modulation of Biointeractions by Electrically Switchable Oligopeptide Surfaces: Structural Requirements and Mechanism. Adv. Mater. Inter. 2014, 1 (2) : 1300085. 
15. Hatakeyama, H.; Kikuchi, A.; Yamato, M.; Okano, T., Bio-functionalized thermoresponsive interfaces facilitating cell adhesion and proliferation. Biomaterials 2006, 27 (29), 5069-5078.

16. Desseaux, S.; Klok, H.-A., Temperature-Controlled Masking/Unmasking of CellAdhesive Cues with Poly(ethylene glycol) Methacrylate Based Brushes. Biomacromolecules 2014, 15, 3859-3865.

17. Wischerhoff, E.; Uhlig, K.; Lankenau, A.; Borner, H. G.; Laschewsky, A.; Duschl, C.; Lutz, J. F., Controlled cell adhesion on PEG-based switchable surfaces. Angew. Chem.-Int. Ed. 2008, 47 (30), 5666-5668.

18. Xia, Y. Q.; Tang, Y.; Wu, H.; Zhang, J.; Li, Z. Y.; Pan, F.; Wang, S. J.; Wang, X. J.; Xu, H.; Lu, J. R., Fabrication of Patterned Thermoresponsive Microgel Strips on Cell-Adherent Background and Their Application for Cell Sheet Recovery. Acs Appl. Mater. Inter. 2017, 9 (2), 1255-1262.

19. Tang, Z.; Akiyama, Y.; Okano, T., Recent Development of Temperature-Responsive Cell Culture Surface Using Poly(N-isopropylacrylamide). J. Pol. Sci., Part B .2014, 52, 917-926.

20. Mandal, K.; Balland, M.; Bureau, L., Thermoresponsive Micropatterned Substrates for Single Cell Studies. Plos One 2012, 7 (5): e37548.

21. Han, L.; Zhang, Y. N.; Lu, X.; Wang, K. F.; Wang, Z. M.; Zhang, H. P., Polydopamine Nanoparticles Modulating Stimuli-Responsive PNIPAM Hydrogels with Cell/Tissue Adhesiveness. Acs Appl. Mater. Inter. 2016, 8 (42), 29088-29100.

22. Yu, Q.; Johnson, L. M.; Lopez, G. P., Nanopatterned Polymer Brushes for Triggered Detachment of Anchorage-Dependent Cells. Adv. Funct. Mater. 2014, 24 (24), 3751-3759.

23. Zahouani, S.; Chaumont, A.; Senger, B.; Boulmedais, F.; Schaaf, P.; Jierry, L.; Lavalle, P., Stretch-Induced Helical Conformations in Poly(L-lysine)/Hyaluronic Acid Multilayers. Acs Appl. Mater. Inter. 2016, 8 (24), 14958-14965.

24. H.-P.Huang; Michel, R.; Vörös, J.; Textor, M.; Hofer, R.; Rossi, A.; Elbert, D. L.; HubbellSpencer, J. A., Poly(L-lysine)-g-poly(ethylene glycol) layers on metal oxide surfaces: surface-analytical characterization and resistance to serum and fibrinogen adsorption. Langmuir 2001, 17, 489-498.

25. Dalier, F.; Eghiaian, F.; Scheuring, S.; Marie, E.; Tribet, C., Temperature-Switchable Control of Ligand Display on Adlayers of Mixed Poly(lysine)-g-(PEO) and Poly(lysine)-g(ligand-modified poly-N-isopropylacrylamide). Biomacromolecules 2016, 17 (5), 1727-1736.

26. van Dongen, S. F. M.; Janvore, J.; van Berkel, S. S.; Marie, E.; Piel, M.; Tribet, C., Reactive protein-repellent surfaces for the straightforward attachment of small molecules up to whole cells. Chem. Sci. 2012, 3 (10), 3000-3006. 
27. Arnold, M.; Schwieder, M.; Blummel, J.; Cavalcanti-Adam, E. A.; Lopez-Garcia, M.; Kessler, H.; Geiger, B.; Spatz, J. P., Cell interactions with hierarchically structured nanopatterned adhesive surfaces. Soft Matter 2009, 5 (1), 72-77.

28. Malinge, J.; Mousseau, F.; Zanchi, D.; Brun, G.; Tribet, C.; Marie, E., Tailored stimuliresponsive interaction between particles adjusted by straightforward adsorption of mixed layers of Poly(lysine)-g-PEG and Poly(lysine)-g-PNIPAM on anionic beads. J. Coll. Inter. Sci. 2016, $461,50-55$. 